https://helda.helsinki.fi

\title{
Exactly solvable model of calorimetric measurements
}

\section{Donvil, Brecht}

2020-12-01

Donvil , B , Muratore-Ginanneschi , P \& Golubev , D 2020 , ' Exactly solvable model of calorimetric measurements ' , Physical Review B , vol. 102 , no. 24 , 245401 . https://doi.org/10.1103/PhysRevB.102

http://hdl.handle.net/10138/324334

https://doi.org/10.1103/PhysRevB.102.245401

unspecified

publishedVersion

Downloaded from Helda, University of Helsinki institutional repository.

This is an electronic reprint of the original article.

This reprint may differ from the original in pagination and typographic detail.

Please cite the original version. 


\title{
Exactly solvable model of calorimetric measurements
}

\author{
Brecht Donvil $\odot$ and Paolo Muratore-Ginanneschi $\odot$ \\ Department of Mathematics and Statistics, University of Helsinki, P.O. Box 68, 00014 Helsinki, Finland \\ Dmitry Golubev $\odot$ \\ Pico group, QTF Centre of Excellence, Department of Applied Physics, Aalto University, P.O. Box 15100, 00076 Aalto, Finland
}

(Received 31 July 2020; revised 13 November 2020; accepted 17 November 2020; published 1 December 2020)

\begin{abstract}
Calorimetric measurements are experimentally realizable methods to assess thermodynamics relations in quantum devices. With this motivation in mind, we consider a resonant level coupled to a Fermion reservoir. We consider a transient process in which the interaction between the level and the reservoir is initially switched on and then switched off again. We find the time dependence of the energy of the reservoir, of the energy of the level, and of the interaction energy between them at weak, intermediate, strong, and ultrastrong coupling. We also determine the statistical distributions of these energies.
\end{abstract}

DOI: 10.1103/PhysRevB.102.245401

\section{INTRODUCTION}

Large, yet finite, reservoirs can simultaneously serve as an environment and a measuring device of the system they are in contact with. Indeed, the energy of the reservoir is influenced by interacting with the system, and by measuring it, one can probe the system [1]. This idea has motivated a series of recent theory papers [2-6], investigating temperature variations in a small metallic particle, caused by photon exchange with a qubit. Here we extend these ideas to an exactly solvable model of a resonant level coupled to a finite-size metallic reservoir, which is the simplest of the well-studied quantum impurity models [7]. In this model, the variations of the reservoir energy occur due to electron jumps between it and the resonant level.

The problem of energy exchange between a microscopic system and the environment has been studied for a long time with the main emphasis being on the heat to work conversion [8-10] and the fluctuation relations [11-16]. Here we take a different perspective, mainly focusing on the variations of the reservoir energy. In fact, we obtain the time-dependent distributions of the reservoir energy, of the energy of the resonant level, and of the interaction energy. We encompass in our analysis the strong-coupling limit, which favors stronger response of the reservoir energy to the changes in the state of the microscopic system. We consider transient behavior of the system assuming that the resonant level and the reservoir become coupled at time $t=0$ and, afterwards, the system relaxes to the steady state in the limit $t \rightarrow \infty$. Some of our predictions may be tested in experiments with quantum dots, which are well described by the resonant level model.

In related works, the weak-coupling regime is typically considered, in which the quantum system is described by a Markovian Lindblad equation [17]. For example, the distribution of energy emitted into the environment by a driven two-level system was previously determined in this regime
$[18,19]$. More recently, the strong-coupling non-Markovian regime has drawn considerable attention. An efficient approximate method of studying strong system-reservoir coupling is the reaction coordinate formalism [20-25], in which the reservoir is replaced by a single degree of freedom weakly coupled to the residual environment. This method ensures quick convergence to the exact result if one increases the number of reaction coordinates [26,27]. The exact strongcoupling dynamics of the energy exchange between a driven two-level system and its environment has been studied within the spin-boson model $[28,29]$. It was found that the strong coupling manifests itself in the significant role of the interaction energy in the overall energy balance $[28,30]$ and in the nonexponential time relaxation of the energies.

The advantage of the resonant level model, which we discuss here, is that it allows one to study the strong-coupling effects in the energy exchange between the system and the environment exactly and, in the wide-band limit, analytically even in the nonstationary case (see, e.g., Ref. [31]). This simple model has been studied for a long time, and its exact solution is presented, for example, in Refs. [32-34]. It has also proven to be very useful in the context of quantum thermodynamics. It has been used, for example, to properly define the thermodynamic quantities of a slowly driven system in the strong-coupling limit [35-38], where the interaction energy cannot be neglected and, therefore, it becomes difficult to separate the heat from the work done on the system. These definitions were verified numerically in Ref. [39], where the fast-driving regime was also studied. Equilibrium fluctuations of the energy of the resonant level were analyzed in Ref. [40]. In addition, the resonant level model becomes equivalent to the so-called Toulouse limit of the spin-boson model if the level is aligned with the Fermi energy of the reservoir [41]. Finally, a comprehensive introduction to fluctuations of thermodynamic quantities in similar models can be found in Ref. [42]. 
This paper is organized as follows: in Sec. II we introduce the model and provide its formal solution in a general form. In Sec. III we apply these results to the specific case of a metallic reservoir with energy-independent spectral density of the environment. In Sec. IV we discuss a possible experimental setup in which our predictions could be tested and summarize our findings.

\section{MODEL}

We consider a spin-polarized resonant energy level coupled to a finite-size reservoir. The total system is described by the Hamiltonian

$$
\hat{H}=\hat{H}_{0}+\hat{H}_{R}+\hat{H}_{I}
$$

Here

$$
\hat{H}_{0}=\epsilon_{0} \hat{c}_{0}^{\dagger} \hat{c}_{0}
$$

is the Hamiltonian of the resonant level,

$$
\hat{H}_{R}=\sum_{k=1}^{\infty} \epsilon_{k} \hat{c}_{k}^{\dagger} \hat{c}_{k}
$$

is the reservoir Hamiltonian, and

$$
\hat{H}_{I}=\sum_{k=1}^{\infty} g_{k}\left(\hat{c}_{0}^{\dagger} \hat{c}_{k}+\hat{c}_{k}^{\dagger} \hat{c}_{0}\right)
$$

is the interaction between them. In addition to the second quantized Hamiltonian (1), we also define the single-particle Hamiltonian of the system

$$
H=H_{0}+H_{R}+H_{I}
$$

where the Hamiltonians $H_{0}, H_{R}$, and $H_{I}$ are infinite-size selfadjoint matrices of the form

$H_{0}=\left(\begin{array}{cc}\epsilon_{0} & \mathbf{0}^{\top} \\ \mathbf{0} & \tilde{0}\end{array}\right), H_{R}=\left(\begin{array}{cc}0 & \mathbf{0}^{\top} \\ \mathbf{0} & \tilde{\epsilon}_{R}\end{array}\right), H_{I}=\left(\begin{array}{cc}0 & \boldsymbol{g}^{\top} \\ \boldsymbol{g} & \tilde{0}\end{array}\right)$.

Here $\top$ stands for transposition, and $\boldsymbol{g}^{\top}=\left(g_{1}, g_{2}, \ldots, g_{N}\right)$ is the row vector containing the coupling constants. All matrix elements of the matrix $\tilde{0}$ and of the vector $\mathbf{0}$ are equal to zero, and $\tilde{\epsilon}_{R}$ is the diagonal matrix containing the energies of the reservoir levels, $\left(\tilde{\epsilon}_{R}\right)_{i j}=\epsilon_{i} \delta_{i j}$. The Hamiltonian $\hat{H}(1)$ can be expressed as

$$
\hat{H}=\hat{\boldsymbol{c}}^{\dagger} H \hat{\boldsymbol{c}},
$$

where $\hat{\boldsymbol{c}}^{\dagger}=\left(\hat{c}_{0}^{\dagger}, \hat{c}_{1}^{\dagger}, \hat{c}_{2}^{\dagger}, \ldots\right)$.

Since the Hamiltonian (1) is quadratic, the Fermions do not interact, and one can infer full information about the energies from the single-particle density matrix of the system $\rho$, which has the matrix elements

$$
\rho_{m n}(t)=\operatorname{tr}\left[\hat{c}_{n}^{\dagger} \hat{c}_{m} \hat{\rho}(t)\right] .
$$

Here $\hat{\rho}(t)$ is the full density matrix of the system in Fock space. The density matrix $\rho$ satisfies the usual Liouville-von Neumann equation

$$
\imath \partial \rho / \partial t=[H, \rho]
$$

with the solution

$$
\rho(t)=e^{-l H t} \rho(0) e^{\imath H t} .
$$

Here $\rho(0)$ is an arbitrary initial density matrix. We restrict our attention to the physically relevant case of a diagonal initial density matrix

$$
\rho_{n k}(0)=n_{k} \delta_{n k},
$$

with populations specified by a Fermi-Dirac thermal equilibrium distribution

$$
n_{k} \equiv n\left(\epsilon_{k}\right)=\frac{1}{1+e^{\left(\epsilon_{k}-\mu\right) / T}} .
$$

Here $\mu$ is the chemical potential of the metallic reservoir, and $T$ is its initial temperature (Boltzmann constant $k_{B}=1$ ).

Due to the simplicity of the model, we can derive an explicit expression for the single-particle evolution operator using the Laplace transform,

$$
e^{-\imath H t}=\left(\begin{array}{cc}
\varphi & f^{\top} \\
\boldsymbol{f} & \tilde{F}
\end{array}\right)
$$

Here $\varphi$ is the occupation amplitude of the resonant level, which is specified by the anti-Laplace transform

$$
\varphi(t)=\int_{\mathcal{B}} \frac{\mathrm{d} z}{2 \pi} \frac{i e^{-l z t}}{z-\epsilon_{0}-\Sigma(z)}
$$

on the (rotated) Bromwich contour

$$
\mathcal{B}=\{z \mid 0<\operatorname{Im} z=\text { const }\} .
$$

$\Sigma(z)$ is the self-energy defined for any $\operatorname{Im} z \neq 0$ by the integral

$$
\Sigma(z)=\int_{\mathbb{R}} \frac{d \epsilon}{2 \pi} \frac{J(\epsilon)}{z-\epsilon},
$$

and

$$
J(\epsilon)=2 \pi \sum_{k=1}^{\infty} g_{k}^{2} \delta\left(\epsilon-\epsilon_{k}\right)
$$

is the environment spectral density. Besides that, we have also introduced the vector $f$ with the elements

$$
f_{k}(t)=-l g_{k} \int_{0}^{t} d t^{\prime} e^{-\imath \epsilon_{k}\left(t-t^{\prime}\right)} \varphi\left(t^{\prime}\right),
$$

and the square subblock $\tilde{F}$ with the matrix elements

$$
\tilde{F}_{n k}(t)=\delta_{n k} e^{-l \epsilon_{k} t}+\frac{f_{n}(t) g_{k}-g_{n} f_{k}(t)}{\epsilon_{n}-\epsilon_{k}} .
$$

The diagonal matrix elements $\tilde{F}_{k k}$ should be obtained from (8) by taking the limit $\epsilon_{n} \rightarrow \epsilon_{k}$.

Having found the single-particle evolution operator (5), we can find the average energy of the resonant level,

$$
\left\langle E_{0}(t)\right\rangle=\operatorname{tr}\left[H_{0} e^{-\imath H t} \rho(0) e^{\imath H t}\right]=\epsilon_{0} \rho_{00}(t),
$$

where the average occupation probability of the level is

$$
\rho_{00}(t)=|\varphi(t)|^{2} n_{0}+\sum_{k=1}^{\infty}\left|f_{k}(t)\right|^{2} n_{k} .
$$

In the same way, the average change in the reservoir energy can be expressed in the 
form

$$
\begin{aligned}
\left\langle\Delta E_{R}(t)\right\rangle & =\operatorname{tr}\left\{H_{R}\left[e^{-\imath H t} \rho(0) e^{\imath H t}-\rho(0)\right]\right\} \\
& =\sum_{k=1}^{\infty} \epsilon_{k}\left[\rho_{k k}(t)-n_{k}\right] .
\end{aligned}
$$

The matrix elements $\rho_{k k}(t)$ can be found from Eq. (5),

$$
\rho_{k k}(t)=\left|f_{k}(t)\right|^{2} n_{0}+\sum_{p=1}^{\infty}\left|F_{k p}(t)\right|^{2} n_{p} .
$$

Invoking the unitarity of the evolution operator (5), we transform Eq. (11) to an alternative form,

$$
\left\langle\Delta E_{R}\right\rangle=\sum_{k=1}^{\infty} \epsilon_{k}\left|f_{k}\right|^{2}\left(n_{0}-n_{k}\right)+\sum_{n, k=1}^{\infty}\left|F_{n k}\right|^{2}\left(\epsilon_{n}-\epsilon_{k}\right) n_{k},
$$

which is preferable for a macroscopic reservoir with a dense spectrum because it is insensitive to the singular behavior of the matrix elements (8) at $n=k$.

The average interaction energy can be inferred from energy conservation,

$$
\left\langle E_{0}(t)\right\rangle+\left\langle E_{R}(t)\right\rangle+\left\langle E_{I}(t)\right\rangle=\epsilon_{0} n_{0}+\sum_{k=1}^{\infty} \epsilon_{k} n_{k},
$$

and Eqs. (9) and (13).

Employing the standard methods of full counting statistics for fermions $[43,44]$, one can derive the statistical distributions of the energies $E_{R}, E_{0}$, and $E_{I}$,

$P_{\alpha}(E, t)=\int \frac{d \nu}{2 \pi} e^{l E v} \operatorname{det}\left[1-\rho(0)+e^{i H t} e^{-i H_{\alpha} v} e^{-l H t} \rho(0)\right]$.

Here the index $\alpha$ can take the values $\alpha=0, R, I$, and the single-particle Hamiltonians $H_{\alpha}$ are defined in Eq. (3).

The simple form of the Hamiltonian $H_{0}$ allows one to find the distribution of the energy of the resonant level exactly,

$$
P_{0}(E, t)=\left[1-\rho_{00}(t)\right] \delta(E)+\rho_{00}(t) \delta\left(E-\epsilon_{0}\right) .
$$

The result (16) implies that the energy of the resonant level randomly jumps between two fixed values: $\epsilon_{0}$, corresponding to the occupied level, and 0 , corresponding to the empty level.

One can also derive an explicit expression for the probability distribution of the interaction energy. The interaction Hamiltonian $H_{I}$ has three eigenvalues: a multiply degenerate eigenvalue 0 and two nondegenerate eigenvalues with opposite signs $\pm \Delta E_{I}$, where

$$
\Delta E_{I}=\sqrt{\sum_{k=1}^{\infty} g_{k}^{2}}
$$

is the "quantum" of the interaction energy. The corresponding eigenvectors have the components

$$
\left|\psi_{ \pm}\right\rangle^{\top}=(1 / \sqrt{2})\left(1, \pm \boldsymbol{g}^{\top} / \Delta E_{I}\right) .
$$

Rewriting the determinant in Eq. (15) in the basis of the eigenvectors of the matrix $H_{I}$, one observes that it reduces to the determinant of a simple $2 \times 2$ matrix. The latter can be evaluated, which results in the following energy distribution:

$$
\begin{aligned}
P_{I}(E, t)= & {\left[1-W_{+}(t)-W_{-}(t)\right] \delta(E)+W_{+}(t) \delta\left(E-\Delta E_{I}\right) } \\
& +W_{-}(t) \delta\left(E+\Delta E_{I}\right) .
\end{aligned}
$$

Here the probabilities $W_{ \pm}(t)$ have the form

$$
\begin{aligned}
& W_{+}(t)=\rho_{++}\left(1-\rho_{--}\right)+\left|\rho_{+-}\right|^{2}, \\
& W_{-}(t)=\rho_{--}\left(1-\rho_{++}\right)+\left|\rho_{+-}\right|^{2},
\end{aligned}
$$

and the matrix elements of the density matrix are defined as

$$
\rho_{s s^{\prime}}(t)=\left\langle\psi_{s}\left|e^{-l H t} \rho(0) e^{l H t}\right| \psi_{s^{\prime}}\right\rangle
$$

and $s, s^{\prime}= \pm$.

The distribution of the reservoir energy cannot be found exactly. However, its general form in the weak-coupling limit can be easily figured out. Indeed, in this case one can ignore the interaction energy $E_{I}$ and apply the energy conservation condition (14) for instantaneous, fluctuating values of the energies $E_{0}$ and $\Delta E_{R}$. This approximation corresponds to the quantum jump approach often used in quantum optics. From Eq. (14) one then obtains $\Delta E_{R}(t)=\epsilon_{0} n_{0}-E_{0}(t)$, and the distribution of the reservoir energy follows from Eq. (16),

$$
\begin{aligned}
P_{R}(\Delta E, t)= & {\left[1-\rho_{00}(t)\right] \delta\left(\Delta E-\epsilon_{0} n_{0}\right) } \\
& +\rho_{00}(t) \delta\left[\Delta E-\epsilon_{0}\left(n_{0}-1\right)\right] .
\end{aligned}
$$

If the energy level is initially occupied, $n_{0}=1$, the distribution (19) has one peak at $\Delta E_{R}=0$ and a second peak at positive energy $\Delta E_{R}=\epsilon_{0}$. In this case, the reservoir energy can either stay unchanged or increase by $\epsilon_{0}$ if an electron leaves the level $\epsilon_{0}$ and enters the reservoir. If the energy level is initially empty, $n_{0}=0$, the peaks of the distribution (19) occur at $\Delta E_{R}=0$ and $\Delta E_{R}=-\epsilon_{0}$. The latter peak describes the reduction in the reservoir energy, which occurs if an electron leaves the reservoir and populates the level $\epsilon_{0}$. If one goes beyond the weak-coupling limit, the $\delta$ peaks in the distribution (19) acquire finite width $\sim\left|\operatorname{Im}\left[\Sigma\left(\epsilon_{0}\right)\right]\right|$.

\section{METALLIC RESERVOIR}

We now apply the general results presented in the previous section to an important example: a metallic reservoir. We thus consider a nonvanishing and constant spectral density

$$
J(\epsilon)=\Gamma_{0} \theta\left(\epsilon_{c}-\epsilon\right) \theta(\epsilon)
$$

in a region delimited by sharp cutoffs at $\epsilon=0$ and at $\epsilon=$ $\epsilon_{c}>0$. The precise value of the cutoff energy $\epsilon_{c}$ is not important because most of the measurable parameters depend on it logarithmically.

\section{A. Long-time asymptotics and general analysis}

The self-energy (7) of the metallic reservoir model becomes

$$
\Sigma(z)=-\frac{\Gamma_{0}}{2 \pi}\left[\ln \left(z-\epsilon_{c}\right)-\ln (z)\right]
$$

for any complex $z$ outside a branch cut in $z \in\left[0, \epsilon_{c}\right]$. When crossing the branch cut from positive to negative values of 


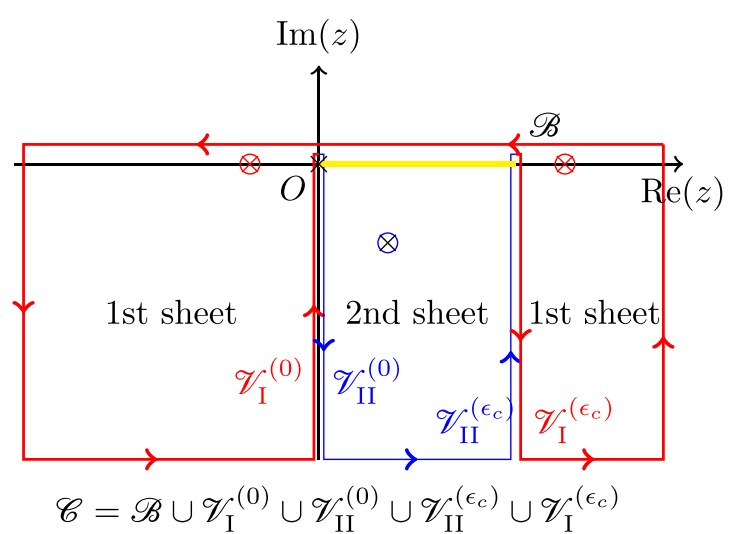

FIG. 1. The contour $\mathcal{C}$ incorporating the Bromwich contour $\mathcal{B}$ of the Laplace antitransform (6) for positive $t . \mathcal{B}, \mathcal{V}_{\mathrm{I}}^{(0)}$, and $\mathcal{V}_{\mathrm{I}}^{\left(\epsilon_{c}\right)}$ (red) are the contour components lying on the first Riemann sheet. $\mathcal{V}_{\mathrm{II}}^{(0)}$ and $\mathcal{V}_{\text {II }}^{\left(\epsilon_{c}\right)}$ (blue) are on the second Riemann sheets. The remaining parts of the contour are at infinity and give vanishing contributions to the occupation amplitude. Circled crosses stand for poles encompassed by the contour.

$\operatorname{Im} z$, the self-energy develops a discontinuity proportional to the intensity of the spectral density

$$
\lim _{\varepsilon \downarrow 0}[\Sigma(x+\imath \epsilon)-\Sigma(x-\imath \epsilon)]=-\imath \Gamma_{0} .
$$

Accordingly, we may analytically extend the self-energy to a second Riemann sheet by requiring continuity across the cut

$$
\Sigma_{\text {II }}(z)=\Sigma(z)-\imath \Gamma_{0} .
$$

In doing so, we also adopt the convention that the principal value $\operatorname{Arg}(z)$ of any complex number $z$ is in the range $(-\pi, \pi]$. These considerations are useful in light of the fact that for any $t>0$ we can most conveniently analyze [33] the integral specifying $\varphi$ by embedding the Bromwich path in the closed contour $\mathcal{C}$ shown in Fig. 1. The contour treads the first and second Riemann sheets. We can then apply Cauchy's theorem to write the occupation probability as

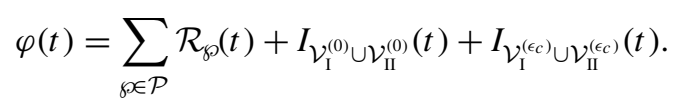

The sum ranges over the residues of the poles $\wp$ enclosed by the contour $\mathcal{C}$, whereas

$$
\begin{aligned}
I_{\mathcal{V}_{\mathrm{I}}^{(0)} \cup \mathcal{V}_{\mathrm{II}}^{(0)}}(t) & =\int_{-\infty}^{0} \frac{d y}{2 \pi} \frac{-\imath \Gamma_{0} e^{y t}}{D(\imath y) D_{\mathrm{II}}(\imath y)}, \\
I_{\mathcal{V}_{\mathrm{I}}^{\left(c_{c}\right)} \cup \mathcal{V}_{\mathrm{II}}^{\left(\epsilon_{c}\right)}}(t) & =\int_{-\infty}^{0} \frac{d y}{2 \pi} \frac{\imath \Gamma_{0} e^{-l \epsilon_{c} t+y t}}{D\left(\epsilon_{c}+\imath y\right) D_{\mathrm{II}}\left(\epsilon_{c}+\imath y\right)},
\end{aligned}
$$

and

$$
D(z)=z-\epsilon_{0}-\Sigma(z), \quad D_{\mathrm{II}}(z)=z-\epsilon_{0}-\Sigma_{\mathrm{II}}(z) .
$$

A detailed study of the analytic properties of the occupation probability integrand [45] shows that poles on the first Riemann sheet can occur only on the real line outside the branch cut. Poles on the first Riemann sheet are thus solutions of

$$
x-\epsilon_{0}+\frac{\Gamma_{0}}{4 \pi} \ln \frac{\left(x-\epsilon_{c}\right)^{2}}{x^{2}}=0
$$

and physically bring about Rabi-like oscillations in the occupation amplitude of the resonant level. Conversely, in the region of the second Riemann sheet enclosed by contour $\mathcal{C}$ in Fig. 1 poles are solutions of the system,

$$
\begin{array}{r}
x-\epsilon_{0}+\frac{\Gamma_{0}}{4 \pi} \ln \frac{\left(x-\epsilon_{c}\right)^{2}+y^{2}}{x^{2}+y^{2}}=0, \\
y+\frac{\Gamma_{0}}{2 \pi}\left(\arctan \frac{y}{x-\epsilon_{c}}-\arctan \frac{y}{x}\right)+\frac{\Gamma_{0}}{2}=0
\end{array}
$$

for

$$
z=x+l y .
$$

In general, residues of poles with a finite imaginary part lead to exponentially decaying contributions to a probability amplitude.

Finally, the contributions of the vertical contours (21) are proportional to $\Gamma_{0}$ at small coupling and to $\Gamma_{0}^{-1}$ at very large coupling. Furthermore, we observe that for finite $t$ the integrands in (21) differ significantly from zero for energies of the order of $1 / t$. Upon expanding the denominators around $1 / t$ we obtain, at leading order, the estimate

$$
\begin{aligned}
& I_{\mathcal{V}_{\mathrm{I}}^{(0)} \cup \mathcal{V}_{\mathrm{II}}^{(0)}}(t)+I_{\mathcal{V}_{\mathrm{I}}^{(\epsilon)} \cup \mathcal{V}_{\mathrm{II}}^{(\epsilon)}}(t) \\
& \quad \approx \frac{\imath \Gamma_{0} e^{-l \epsilon_{c} t}}{t D\left(\epsilon_{c}+\imath / t\right) D_{\mathrm{II}}\left(\epsilon_{c}+\imath / t\right)}-\frac{\imath \Gamma_{0}}{t D(l / t) D_{\mathrm{II}}(l / t)} .
\end{aligned}
$$

The accuracy of the estimate improves as time elapses (see Fig. 4 below). For

$$
t \gg \frac{1}{\epsilon_{c}} e^{\frac{3 \pi}{2}}, \quad \frac{1}{\epsilon_{c}} e^{\frac{2 \pi\left|\epsilon_{c}-\epsilon_{0}\right|}{\Gamma_{0}}},
$$

the estimate (24) reduces to the simpler expression

$$
\begin{aligned}
& I_{\mathcal{V}_{\mathrm{I}}^{(0)} \cup \mathcal{V}_{\mathrm{II}}^{(0)}}(t)+I_{\mathcal{V}_{\mathrm{I}}^{\left(\epsilon_{c}\right)} \cup \mathcal{V}_{\mathrm{II}}^{\left(\epsilon_{c}\right)}}(t) \\
& \stackrel{t \uparrow \infty}{\rightarrow} \frac{4 \pi e^{-t \frac{\epsilon_{c t}}{2}}}{\Gamma_{0} t \ln ^{2}\left(\epsilon_{c} t\right)} \sin \left(\frac{\epsilon_{c} t}{2}\right) .
\end{aligned}
$$

The derivation of further analytic asymptotic expressions hinges upon the introduction of explicit assumptions on the strength of the coupling.

\section{B. Numerical analysis}

We numerically compute the evolution operator (5) by direct exponentiation of the single-particle Hamiltonian (2) for a finite amount of energy levels $N$ in the reservoir. We keep the level spacing in the reservoir constant $\Delta \omega=\epsilon_{c} / N$ and take coupling constants to be

$$
g_{k}=\sqrt{\frac{\Gamma_{0} \Delta \omega}{2 \pi}} .
$$

Note that we scale the coupling with the level spacing $\Delta \omega$ such that the total coupling does increase with increasing reservoir size [46]. Having computed (5), we are able to evaluate Eqs. (9) and (13) for the energy of the resonant level and the change in reservoir energy.

To obtain the full probability distributions, we take a slightly different route and directly evaluate Eq. (15). We compute the determinant by numerically exponentiating the 
matrices. Finally, we perform the Fourier transform using the fast Fourier transform algorithm.

\section{Weak- and intermediate-coupling asymptotic analysis}

We assume the following relation between the parameters:

$$
\Gamma_{0}, T \ll \mu, \quad \epsilon_{0} \ll \epsilon_{c} .
$$

The relation between $\Gamma_{0}$, the temperature $T$, and the detuning between the level and the Fermi energy, $\left|\epsilon_{0}-\mu\right|$, can be arbitrary. The condition (26) covers most physically relevant situations, in which a metallic reservoir with big Fermi energy is involved. It also includes the regime of intermediate coupling, where the deviations from Markovian dynamics already become significant.

In this regime, residues of the poles on the first Riemann sheet do not give any sizable contribution. Although (22) admits two roots outside the branch cut for any positive $\Gamma_{0}$, such roots emerge from the branch cut end points with a nonanalytic dependence upon $\Gamma_{0}$ :

$$
x_{-} \sim-\epsilon_{c} e^{-2 \pi \frac{\epsilon_{0}}{\Gamma_{0}}}, \quad x_{+} \sim \epsilon_{c}+\epsilon_{c} e^{-2 \pi \frac{\epsilon_{c}-\epsilon_{0}}{\Gamma_{0}}},
$$

and the corresponding residues are exponentially suppressed.

On the second Riemann sheet (23) admits, within leadingorder accuracy in the coupling, the solution

$$
z_{*}=\tilde{\epsilon}_{0}+\imath \frac{\Gamma_{0}}{2} .
$$

The quantity

$$
\tilde{\epsilon}_{0}=\epsilon_{0}-\frac{\Gamma_{0}}{2 \pi} \ln \left|\frac{\epsilon_{c}-\epsilon_{0}}{\epsilon_{0}}\right|
$$

physically describes the energy of the resonant level shifted due to its coupling to the reservoir. Upon evaluating the residue of (27) and recalling that the contributions (21) are proportional to $\Gamma_{0}$ at small coupling, within leading accuracy we get

$$
\begin{aligned}
\varphi(t) & =e^{-i \tilde{\epsilon}_{0} t} e^{-\Gamma_{0} t / 2}, \\
f_{k}(t) & =g_{k} \frac{e^{-i \epsilon_{k} t}-e^{-i \tilde{\epsilon}_{0} t} e^{-\Gamma_{0} t / 2}}{\epsilon_{k}-\tilde{\epsilon}_{0}+i \frac{\Gamma_{0}}{2}} .
\end{aligned}
$$

The self-consistency condition for (28) is

$$
t \ll t_{K}
$$

where $t_{K}$ is the timescale predicted by Khalfin's theorem after which unitary dynamics forbids exponential decay [47]. We estimate $t_{K}$ by matching exponential decay with the involution of the longtime asymptotics (25)

$$
e^{-\Gamma_{0} t_{K} / 2} \sim \frac{4 \pi}{\Gamma_{0} t_{K} \ln ^{2}\left(\epsilon_{c} t_{K}\right)} .
$$

In particular, if model parameters are as in Fig. 2, we find that for $\Gamma_{0}=0.001 \mu$ Khalfin's time is $\Gamma_{0} t_{K} \approx 9.1$, whereas for $\Gamma_{0}=0.02 \mu$ we get $\Gamma_{0} t_{K} \approx 7.4$. In both cases $e^{-\Gamma_{0} t_{K}} \ll$ 1 , which indicates that one can use the approximation (28) during the whole relaxation process. For small long-time tails one should use a better approximation, which was outlined in Sec. III A.
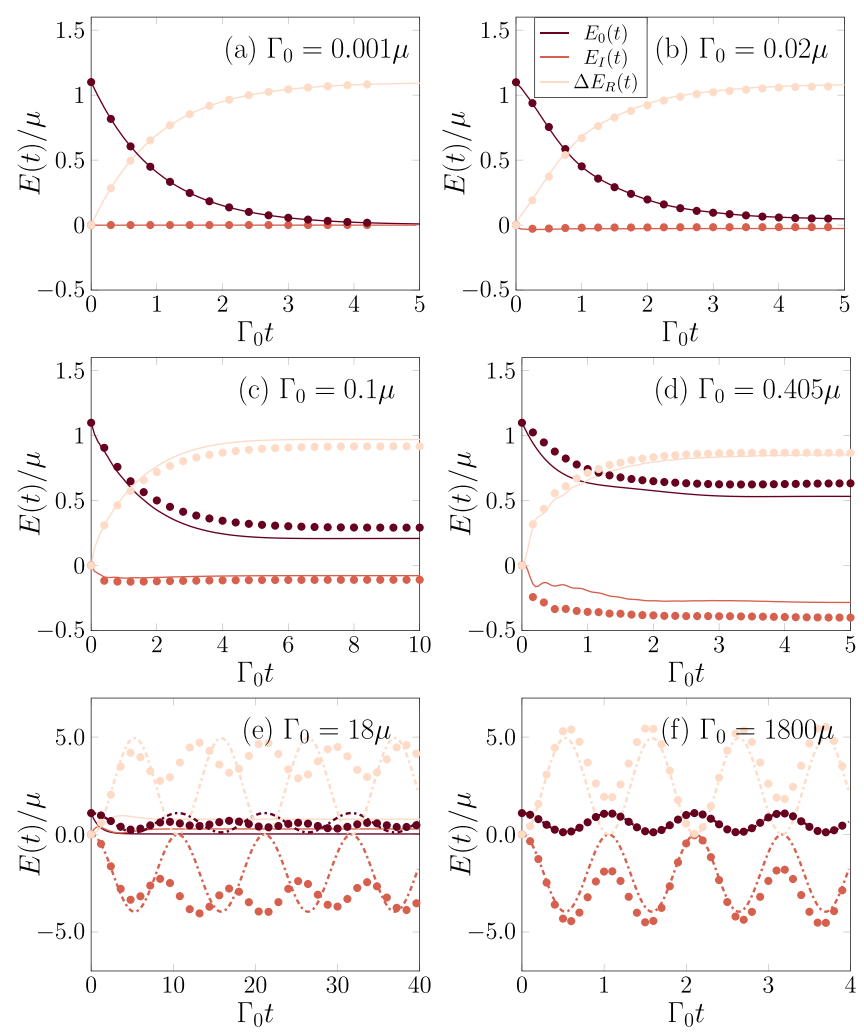

FIG. 2. Time dependence of the energies $E_{0}(t)$ and $\Delta E_{R}(t)$ at (a) and (b) weak, (c) and (d) intermediate, (e) strong, and (f) ultrastrong coupling. The dots are obtained by numerical evaluation of the exact time evolution (5). The solid lines show the analytical weakcoupling predictions (31), and the dashed lines show the ultrastrong coupling predictions (40) and (41). We have assumed $T=0, n_{0}=1$, $\epsilon_{0}=1.1 \mu$, and $\epsilon_{c}=10 \mu$. For (a) the number of modes in the reservoir is $N=7000$, and the level spacing $\Delta \omega=\epsilon_{c} / N=1 / 700 \mu$; for (b), $N=500, \Delta \omega=1 / 50 \mu$; and for (c)-(f) $N=200, \Delta \omega=1 / 20 \mu$.

The occupation probability of the level (10) becomes

$$
\begin{aligned}
\rho_{00}= & e^{-\Gamma_{0} t} n_{0} \\
& +\Gamma_{0} \int_{0}^{\epsilon_{c}} \frac{d \epsilon}{2 \pi} \frac{1+e^{-\Gamma_{0} t}-2 e^{-\frac{\Gamma_{0} t}{2}} \cos \left[\left(\epsilon-\tilde{\epsilon}_{0}\right) t\right]}{\left(\epsilon-\tilde{\epsilon}_{0}\right)^{2}+\frac{\Gamma_{0}^{2}}{4}} n_{F}(\epsilon),
\end{aligned}
$$

where $n_{F}(\epsilon)$ is the Fermi function (4), now evaluated over the continuous variable $\epsilon$. The occupation probabilities of the levels in the reservoir (12) become

$$
\begin{aligned}
\rho_{k k}= & n_{k}+\frac{1+e^{-\Gamma_{0} t}-2 e^{-\frac{\Gamma_{0} t}{2}} \cos \left[\left(\epsilon_{k}-\tilde{\epsilon}_{0}\right) t\right]}{\left(\epsilon_{k}-\tilde{\epsilon}_{0}\right)^{2}+\frac{\Gamma_{0}^{2}}{4}}\left[n_{0}-n_{k}\right] \\
& +g_{k}^{2} \int_{0}^{\epsilon_{c}} \frac{d \epsilon}{2 \pi} \frac{\Gamma_{0}\left[n_{F}(\epsilon)-n_{k}\right]}{\left(\epsilon-\epsilon_{k}\right)^{2}} \\
& \times\left|\frac{e^{-i \epsilon t}-e^{-i \tilde{\epsilon}_{0} t} e^{-\frac{\Gamma_{0} t}{2}}}{\epsilon-\tilde{\epsilon}_{0}+i \frac{\Gamma_{0}}{2}}-\frac{e^{-i \epsilon_{k} t}-e^{-i \tilde{\epsilon}_{0} t} e^{-\frac{\Gamma_{0} t}{2}}}{\epsilon_{k}-\tilde{\epsilon}_{0}+i \frac{\Gamma_{0}}{2}}\right|^{2}
\end{aligned}
$$

The average value of the energy of the resonant level $\left\langle E_{0}(t)\right\rangle$ is given by Eq. (9) in which $\rho_{00}(t)$ has the form (29). Substituting expression (30) in Eq. (13) and replacing the 
summation over $k$ by the integral over the energy $\epsilon_{k}$, we obtain the average change in the reservoir energy in the form

$$
\begin{aligned}
& \frac{\left\langle\Delta E_{R}(t)\right\rangle}{\Gamma_{0}} \\
& =\int_{0}^{\epsilon_{c}} \frac{d \epsilon}{2 \pi}\left[\frac{\epsilon\left[n_{0}-n_{F}(\epsilon)\right]+\frac{\Gamma_{0}}{2 \pi} \ln \frac{\epsilon_{c}-\epsilon_{0}}{\epsilon_{0}} n_{F}(\epsilon)}{\left(\epsilon-\tilde{\epsilon}_{0}\right)^{2}+\frac{\Gamma_{0}^{2}}{4}}\right. \\
& \quad \times\left\{1+e^{-\Gamma_{0} t}-2 e^{-\Gamma_{0} t / 2} \cos \left[\left(\epsilon-\tilde{\epsilon}_{0}\right) t\right]\right\} \\
& \left.\quad-\frac{\left(1-e^{-\Gamma_{0} t}\right)\left(\epsilon-\tilde{\epsilon}_{0}\right)-\Gamma_{0} e^{-\Gamma_{0} t / 2} \sin \left[\left(\epsilon-\tilde{\epsilon}_{0}\right) t\right]}{\left(\epsilon-\tilde{\epsilon}_{0}\right)^{2}+\frac{\Gamma_{0}^{2}}{4}} n_{F}(\epsilon)\right] .
\end{aligned}
$$

The average value of the interaction energy $\left\langle E_{I}(t)\right\rangle$ can be inferred from the energy conservation (14).

In the weak-coupling regime

$$
\Gamma_{0} \ll\left(\tilde{\epsilon}_{0}-\mu\right) \operatorname{coth} \frac{\tilde{\epsilon}_{0}-\mu}{2 T},
$$

the integrals (29) and (31) can be straightforwardly evaluated. We obtain simple exponential relaxation of the energies, which is a typical feature of the Markovian Lindblad approximation,

$$
\begin{aligned}
\left\langle E_{0}(t)\right\rangle & =e^{-\Gamma_{0} t} \epsilon_{0} n_{0}+\left(1-e^{-\Gamma_{0} t}\right) \epsilon_{0} n_{F}\left(\epsilon_{0}\right), \\
\left\langle\Delta E_{R}(t)\right\rangle & =\left(1-e^{-\Gamma_{0} t}\right) \epsilon_{0}\left[n_{0}-n_{F}\left(\epsilon_{0}\right)\right] .
\end{aligned}
$$

The interaction energy in this regime is negligible.

In the long-time limit $t_{K} \gg t \gg(\pi T)^{-1}$ the occupation probabilities of the levels (29) and (30) approach their asymptotic values

$$
\begin{aligned}
\rho_{00}^{\mathrm{as}}= & \frac{1}{2}-\frac{1}{\pi} \operatorname{Im}\left[\Psi\left(\frac{1}{2}+\frac{\Gamma_{0}}{4 \pi T}+\imath \frac{\tilde{\epsilon}_{0}-\mu}{2 \pi T}\right)\right], \\
\rho_{k k}^{\mathrm{as}}= & n_{k}+\frac{g_{k}^{2}}{\left(\epsilon_{k}-\tilde{\epsilon}_{0}\right)^{2}+\frac{\Gamma_{0}^{2}}{4}}\left\{n_{0}+\rho_{00}^{\mathrm{as}}-2 n_{k}\right. \\
& +\frac{\Gamma_{0}}{\pi} \operatorname{Re}\left[\frac{\Psi\left(\frac{1}{2}+\frac{\Gamma_{0}}{4 \pi T}+\imath \frac{\tilde{\epsilon}_{0}-\mu}{2 \pi T}\right)-\Psi\left(\frac{1}{2}+\imath \frac{\epsilon_{k}-\mu}{2 \pi T}\right)}{\epsilon_{k}-\tilde{\epsilon}_{0}+i \frac{\Gamma_{0}}{2}}\right] \\
& \left.-\frac{\Gamma_{0}}{2 \pi^{2} T} \operatorname{Im}\left[\Psi^{\prime}\left(\frac{1}{2}+\imath \frac{\epsilon_{k}-\mu}{2 \pi T}\right)\right]\right\},
\end{aligned}
$$

where $\Psi(x)$ is the digamma function. Accordingly, the energies in the long-time limit take the form

$$
\begin{gathered}
\left\langle E_{0}^{\mathrm{as}}\right\rangle=\epsilon_{0} \rho_{00}^{\mathrm{as}}, \\
\left\langle\Delta E_{R}^{\mathrm{as}}\right\rangle=\epsilon_{0}\left[n_{0}-\rho_{00}^{\mathrm{as}}\right]-\left\langle E_{I}^{\mathrm{as}}\right\rangle, \\
\left\langle E_{I}^{\mathrm{as}}\right\rangle=-\frac{\Gamma_{0}}{\pi}\left\{\ln \frac{\epsilon_{c}-\epsilon_{0}}{\epsilon_{0}} \rho_{00}^{\mathrm{as}}+\ln \frac{\epsilon_{0}}{2 \pi T}\right. \\
\left.-\operatorname{Re}\left[\Psi\left(\frac{1}{2}+\frac{\Gamma_{0}}{4 \pi T}+\imath \frac{\tilde{\epsilon}_{0}-\mu}{2 \pi T}\right)\right]\right\} .
\end{gathered}
$$

The asymptotic distribution function in the metallic reservoir (33) has a Lorentzian peak or dip close to $\tilde{\epsilon}_{0}$. Clearly, such a strong nonequilibrium distribution will relax to the thermal one during the electron-electron relaxation time $\tau_{e-e}$. Thus, the distribution (33) survives only during the time interval $(\pi T)^{-1}<t<\tau_{e-e}$. This condition provides the range of validity of our model.

\section{Ultrastrong-coupling asymptotic analysis}

We now assume that $\Gamma_{0}$ sets the largest energy scale in the model:

$$
\epsilon_{0}, \epsilon_{c} \ll \Gamma_{0} .
$$

In this case, the residues of poles in the first and second Riemann sheets exchange roles in relation to their significance for the dynamics. Namely, (22) admits two solutions,

$$
x_{ \pm}= \pm \Omega_{1}+\Omega_{2}+O\left(\Gamma_{0}^{-1}\right)
$$

with

$$
\begin{aligned}
& \Omega_{1}=\sqrt{\frac{\Gamma_{0} \epsilon_{c}}{2 \pi}}+\sqrt{\frac{2 \pi}{\Gamma_{0}}} \frac{7 \epsilon_{c}^{2}+12 \epsilon_{0}\left(\epsilon_{0}-\epsilon_{c}\right)}{96 \sqrt{\epsilon_{c}}}, \\
& \Omega_{2}=\frac{2 \epsilon_{0}+\epsilon_{c}}{4}
\end{aligned}
$$

appearing to the right $\left(x_{+}\right)$and to the left $\left(x_{-}\right)$of the branch cut. These solutions correspond to two simple poles with residues $\mathcal{R}_{ \pm}$now giving an $O(1)$ contribution to (20):

$$
\begin{aligned}
\mathcal{R}_{+}+\mathcal{R}_{-}= & e^{-\imath \Omega_{2} t}\left(1-\frac{\pi \epsilon_{c}}{6 \Gamma_{0}}\right) \cos \left(\Omega_{1} t\right) \\
& +\imath e^{-\imath \Omega_{2} t} \sqrt{\pi} \frac{\epsilon_{c}-2 \epsilon_{0}}{\sqrt{8 \epsilon_{c} \Gamma_{0}}} \sin \left(\Omega_{1} t\right)+O\left(\Gamma_{0}^{-3 / 2}\right) .
\end{aligned}
$$

Note that the energy $\Omega_{1}$ approaches the interaction energy quantum (17) in the limit of infinitely strong coupling, $\Omega_{1}=$ $\Delta E_{I}$ for $\Gamma_{0} \rightarrow \infty$. On the second Riemann sheet, (23) admits the solution

$$
z_{\star}=\epsilon_{0}+\imath \frac{\Gamma_{0}}{2}+O\left(\Gamma_{0}^{-1}\right) .
$$

The imaginary part of the root entails an exponential suppression of the residue with a very large rate. As a consequence, the contribution to (20) is negligible after an elapse of any nonvanishing time interval $t$. Finally, (24) estimates the contribution of the vertical tracts of the complex plane contour $\mathcal{C}$ as being of the order of $\left(\Gamma_{0} t\right)^{-1}$ for finite $t$. The upshot is that energy statistics within leading-order accuracy are dominated by stable oscillations determined by the residues of the first Riemann sheet poles (37). Indeed,

$$
\begin{gathered}
\varphi(t)=e^{-\imath \Omega_{2} t} \cos \left(\Omega_{1} t\right)+O\left(\Gamma_{0}^{-1 / 2}\right), \\
f_{k}(t)=-l g_{k} \frac{e^{-\imath \Omega_{2} t}}{\Omega_{1}} \sin \left(\Omega_{1} t\right)\left[1+O\left(\Gamma_{0}^{-1 / 2}\right)\right]
\end{gathered}
$$

yield [up to corrections $O\left(\Gamma_{0}^{-1 / 2}\right)$ ]

$$
\begin{aligned}
\left\langle E_{0}(t)\right\rangle= & \epsilon_{0} n_{0} \cos ^{2}\left(\Delta E_{I} t\right) \\
& +\epsilon_{0} \sin ^{2}\left(\Delta E_{I} t\right) \int_{0}^{\epsilon_{c}} \frac{d \epsilon}{\epsilon_{c}} n_{F}(\epsilon)
\end{aligned}
$$

and

$$
\left\langle\Delta E_{R}(t)\right\rangle=\sin ^{2}\left(\Delta E_{I} t\right) \int_{0}^{\epsilon_{c}} \frac{d \epsilon}{\epsilon_{c}} \epsilon[n(0)-n(\epsilon)] .
$$


In this case, the reservoir can effectively be replaced by a single energy level in accordance with the theory of fermionic reaction coordinates [25] and results from the spectral analysis of the Hamiltonian operator where, at strong coupling, one finds that the one-particle Hamiltonian gains a pure point spectrum (see, e.g., $[34,48]$ ).

\section{DISCUSSION}

In Fig. 2(a) we illustrate the exponential time dependence of the energies $\left\langle E_{0}(t)\right\rangle$ and $\left\langle\Delta E_{R}(t)\right\rangle$ at zero temperature and at weak coupling. We assumed that the resonant level was initially populated, $n_{0}=1$. For the chosen parameters, namely, $\Gamma_{0}=0.001 \mu, \epsilon_{0}=1.1 \mu$, and $\epsilon_{c}=10 \mu$, the full solution (29) and (31) and the weak-coupling approximation (32) produce overlapping curves. The dots in Fig. 2(a) are the result of numerical evaluation of the exact dynamics (5) as described in Sec. III B. We find that the numerics quantitatively validate the asymptotic analysis, as expected in this parametric range.

In Figs. 2(b)-2(f) we show the time dependence of the reservoir energy (31), the energy of the resonant level $\left\langle E_{0}(t)\right\rangle$, and the interaction energy at $T=0$, with the same values of $\epsilon_{0}$ and $\epsilon_{c}$, but at stronger coupling. Strong coupling manifests itself in two ways: (i) the interaction energy $\left\langle E_{I}(t)\right\rangle$ becomes comparable to $\left\langle\Delta E_{R}(t)\right\rangle$ and $\left\langle E_{0}(t)\right\rangle$, and (ii) oscillatory contributions to the average energies $\propto e^{-\Gamma_{0} t / 2} \cos \left[\left(\tilde{\epsilon}_{0}-\right.\right.$ $\mu) t]$ become visible. We consider four regimes of systemreservoir coupling as discussed in [45]: weak $\left(\Gamma_{0}=0.02 \mu\right)$, intermediate $\left(\Gamma_{0}=0.1 \mu\right.$ and $\left.0.405 \mu\right)$, strong $\left(\Gamma_{0}=18 \mu\right)$, and ultrastrong $\left(\Gamma_{0}=1800 \mu\right)$ coupling. For $\Gamma_{0}=0.02 \mu$ the energies still display almost exponential decay, and the numerics (dots) and analytical predictions (29) and (31) (solid lines) agree quite well. In the intermediate regime, $\Gamma_{0}=$ $0.1 \mu$ and $0.405 \mu$, deviations from the exponential decay and the oscillations become visible. In addition, at short times the analytic results do not agree with the numerics because the condition (26) is no longer valid. At strong coupling we observe strong oscillations, which do not decay in time, in qualitative agreement with our asymptotic analysis (40) and (41) and results from spectral analysis (see, e.g., [34,48]). Finally, at ultrastrong coupling we again observe strong oscillations with the frequency $\Delta E_{I}$, in agreement with Eqs. (40) and (41).

Next, we plot the long-time asymptotic energies (34)-(36) in Fig. 3. The asymptotic energy of the resonant level (34) is independent of its initial population $n_{0},\left\langle E_{0}^{\text {as }}\right\rangle \approx \tilde{\epsilon}_{0}$ for $\tilde{\epsilon}_{0}<\mu$ and $\left\langle E_{0}^{\text {as }}\right\rangle \approx 0$ for $\tilde{\epsilon}_{0}>\mu$. In contrast, the asymptotic energy of the reservoir is very sensitive to $n_{0}$. For $n_{0}=1$ this energy grows as $\left\langle\Delta E_{R}^{\text {as }}\right\rangle \approx \epsilon_{0}$ for $\tilde{\epsilon}_{0}>\mu$ since in this case the electron escapes from the level to one of the unoccupied states in the reservoir. For $\tilde{\epsilon}_{0}<\mu$ the electron stays on the atom since the Pauli principle prevents it from jumping to the occupied states in the reservoir; hence, $\left\langle\Delta E_{R}^{\text {as }}\right\rangle$ is small. With similar arguments, one can easily understand that for $n_{0}=0$ the reservoir energy should behave as $\left\langle\Delta E_{R}^{\text {as }}\right\rangle \approx-\tilde{\epsilon}_{0}$ for $\tilde{\epsilon}_{0}<\mu$ and $\left\langle\Delta E_{R}^{\text {as }}\right\rangle \approx 0$ for $\tilde{\epsilon}_{0}>0$. In the vicinity of the Fermi level the switching from one regime to another occurs within the interval $\left|\tilde{\epsilon}_{0}-\mu\right| \lesssim \Gamma_{0}$. The average interaction energy (36) is always negative, $\left\langle E_{I}^{\text {as }}\right\rangle \sim-\Gamma_{0}$. In Fig. 3 we have chosen rather weak coupling, $\Gamma_{0}=0.05 \mu$; therefore, the an-
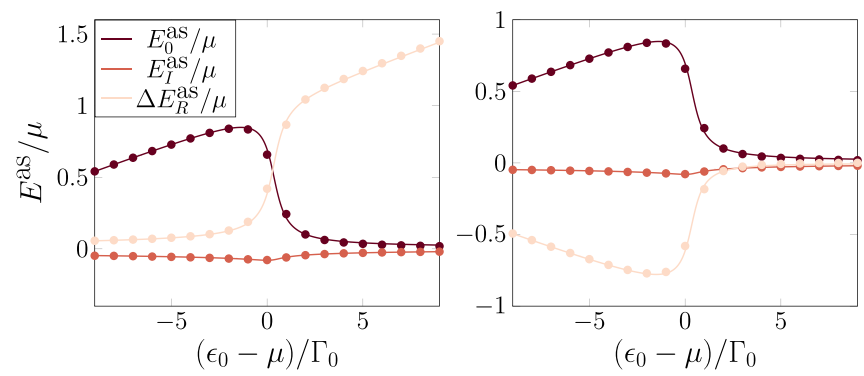

FIG. 3. Average values of the long-time asymptotic energies of the resonant level $\left\langle E_{0}^{\mathrm{as}}\right\rangle$ (34), of the reservoir $\left\langle\Delta E_{R}^{\mathrm{as}}\right\rangle$ (35), and of the interaction energy $\left\langle E_{I}^{\text {as }}\right\rangle$ (36) plotted versus the energy of the level $\epsilon_{0}$. The dots are obtained by numerical evaluation of the exact dynamics (5). The temperature is zero, $T=0$. In the left panel we assume $n_{0}=$ 1 ; that is, the energy level is initially populated. In the right panel we put $n_{0}=0$. The coupling rate between the level and the reservoir is $\Gamma_{0}=0.05 \mu, \epsilon_{c}=10 \mu, N=200$, and $\Delta \omega=1 / 20 \mu$.

alytical expressions (34)-(36) agree with the exact numerics quite well.

In Fig. 4 we show the long-time behavior of the resonant level occupation $|\varphi(t)|^{2}$ for $t$ longer then the Khalfin time $t_{K}$. After a long period of exponential decay, the level enters a regime of power law decay with oscillations.

Finally, Fig. 5 shows the probability distribution of the resonant level, the reservoir, and the interaction energies evaluated numerically. As expected, the distribution of the level energy (16) has two $\delta$ peaks at $E_{0}=0$ and $E_{0}=\epsilon_{0}$. Since we have chosen the initial condition $n_{0}=1$, in the weak-coupling regime the distribution of the reservoir energy also has two peaks centered around $\Delta E_{R}=0$ and $\Delta E_{R}=$ $\epsilon_{0}$. The peak at $\Delta E_{R}=0$ always remains sharp, while the

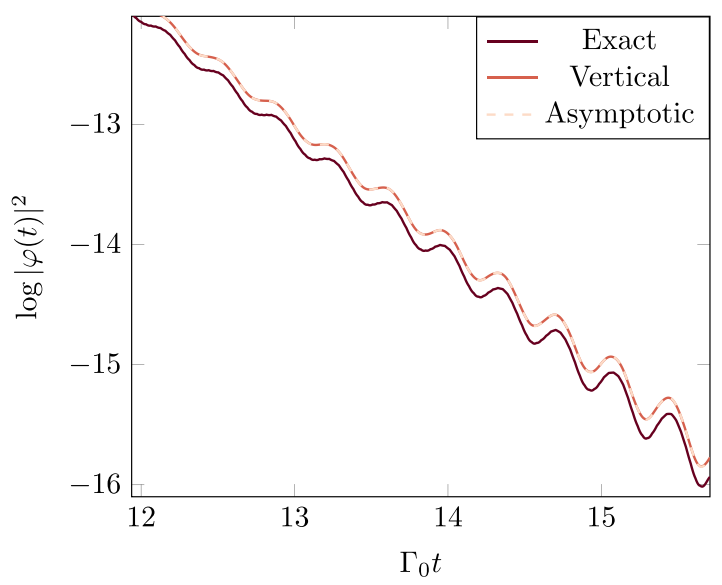

FIG. 4. Long-time nonexponential behavior of $|\varphi(t)|^{2}$ for $\Gamma_{0}=$ $0.0623 \mu, \epsilon_{c}=10 \mu$, and $\epsilon_{0}=1.1 \mu$ : there are three curves, with two overlapping. The dark red curve shows the numerical evaluation of the integral (6). The orange solid line and yellow dashed respectively show the numerical evaluation of the vertical contour contribution plus the residue of (27) and asymptotic evaluation (24) of the vertical contour integral plus the residue contribution. The small discrepancy between the three curves originates from the residues' contributions. The timescales in the plot require retaining all terms in the analytic asymptotic, as reported in the second line of (24). 

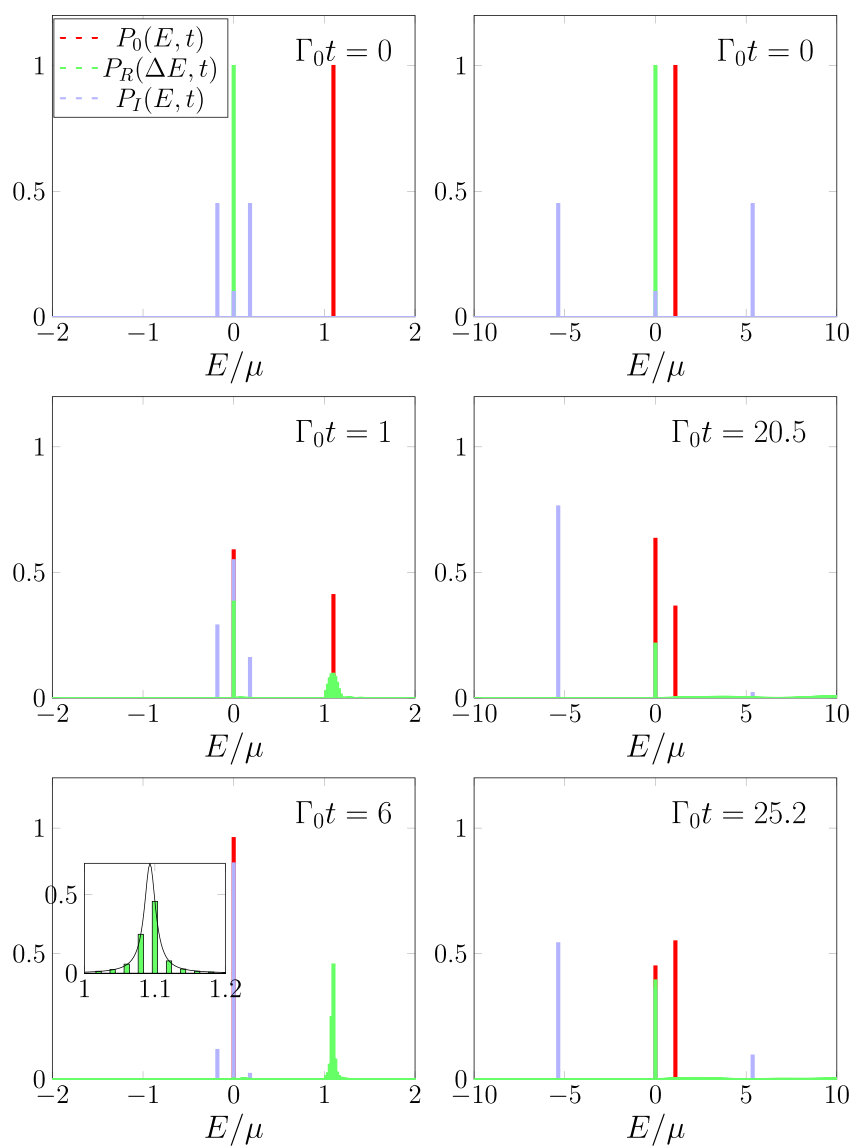

FIG. 5. Probability distributions of the reservoir, interaction, and resonant level energies. The left column corresponds to the weakcoupling regime with $\Gamma_{0}=0.02 \mu$, and the right one corresponds to the strong-coupling limit $\Gamma_{0}=18$. Other parameters have the values $T=0, n_{0}=1, \epsilon=1.1 \mu, \epsilon_{c}=10 \mu, N=200$, and $\Delta \omega=1 / 20 \mu$. The inset in the bottom left panel shows a Lorentzian fit of the peak in the reservoir energy distribution, $P_{R}(\Delta E, t)=a /\left[\left(\Delta E-\tilde{\epsilon}_{0}\right)^{2}+\right.$ $\left.\Gamma_{0}^{2} / 4\right]$. We find from the fit $\Gamma_{0}=0.0193 \mu$, which is close to the expected value $0.02 \mu$.

second peak at $\Delta E_{R}=\epsilon_{0}$ acquires finite length $\sim \Gamma_{0}$ with growing time. In fact, one can show that at long times in the weak-coupling regime this peak should approach the Lorentzian shape $P_{R}(E, t) \propto \Gamma_{0} /\left[\left(\Delta E_{R}-\tilde{\epsilon}_{0}\right)^{2}+\Gamma_{0}^{2} / 4\right]$. Note that in the strong-coupling regime the energy distribution of the reservoir does not show a second peak. Finally, in agreement with Eq. (18), the distribution of the interaction energy has three sharp peaks separated by the intervals (17) $\Delta E_{I}=\sqrt{\Gamma_{0} \epsilon_{c} / 2 \pi}$.

\section{Possible experiment}

In the previous section we demonstrated that the strongcoupling between a resonant energy level and a metallic reservoir can lead to the nonexponential relaxation of the energy and to a significant part of the energy being stored in the interaction part of the Hamiltonian. In this section we will briefly discuss a possible experiment in which these predictions may be tested. We do not aim at a detailed experimental proposal with realistic parameters; rather, we limit

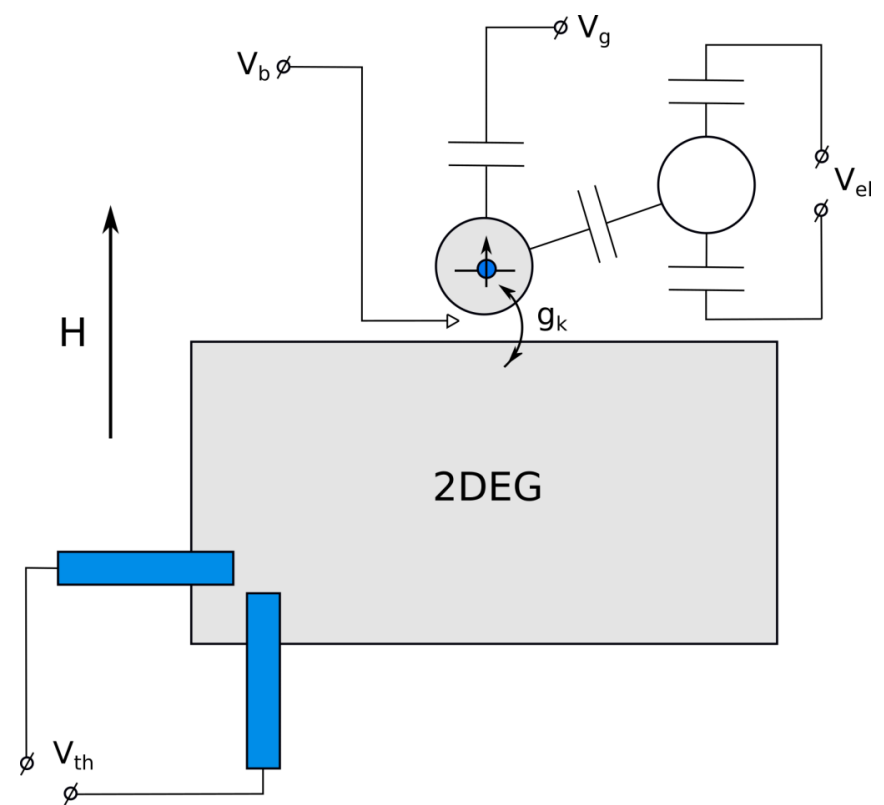

FIG. 6. Sketch of a possible experimental setup. The control potential $V_{b}$ allows one to tune the height of the barrier between the quantum dot hosting a spin-polarized energy level and the 2DEG reservoir, the gate potential $V_{g}$ tunes the position of the level relative to the Fermi energy of the reservoir, the electrometer allows one to monitor the number of electrons in the dot, and the thermometer in the bottom left corner measures the temperature of the reservoir.

ourselves to a qualitative level discussion of a possible experimental protocol and relevant timescales in nanoelectronic devices.

A possible setup for such an experiment would be a system with a finite-size area containing a two-dimensional electron gas (2DEG) playing the role of the metallic reservoir and a quantum dot with an energy level spin polarized by the strong in-plane magnetic field. This setup is depicted in Fig. 6. The charging energy of the dot should be small, $E_{C} \ll \Gamma_{0}$. The barrier between the quantum dot and the reservoir may be tuned by applying the potential $V_{b}$ to the control gate electrode; the position of the level relative to the Fermi energy can be tuned by the gate voltage $V_{g}$, and the number of electrons in the dot can be detected by an electrometer. Finally, the temperature of the 2DEG can be monitored, for example, by a thermometer based on a superconductor-normal metal-superconductor Josephson junction [49]. The experiment should be run as follows: (i) At time $t=0$ the barrier between the dot and the reservoir is reduced, and they become coupled at a rate $\Gamma_{0}$. (ii) At the final time $t$ this coupling is switched off again, and the reservoir is left to relax. (iii) The number of electrons in the dot is measured, and (iv) after the delay time, which should be longer than the electron-electron relaxation time $\tau_{e-e}$ but shorter than the electron-phonon time $\tau_{e-p h}$, the temperature of the 2DEG reservoir is measured. The measured temperature can be converted to the energy of the reservoir as $\Delta E_{R}=C_{V} \Delta T_{R}$, where $C_{V}$ is the heat capacity of the reservoir, and the number of electrons in the dot can be converted into the energy of the resonant level. Corresponding energy distributions can be obtained by repeating this experiment many 
times. The interaction energy cannot be directly measured, but it should be possible to infer its average value from the energy conservation condition (14). As for the original motivation of our study, one should be able to easily determine the initial population of the quantum dot level by measuring the temperature of the reservoir if the energy of the level $\epsilon_{0}$ is sufficiently high. Easily achievable values $\epsilon_{0}>100 \mu \mathrm{eV}$ should be sufficient for that.

This type of experiment is certainly challenging because of the low values of the electron-electron relaxation time $\tau_{e-e}$. Indeed, $\tau_{e-e}$ lies in the nanosecond range at the lowest accessible temperatures [50,51]. This leaves little room for observation of nonexponential time relaxation and long-time asymptotics (24). We believe, however, that one should be able to measure the asymptotic values of the average level and reservoir energies (34) and (35) and subsequently estimate the interaction energy (36) from the conservation condition
(14). The interaction energy should be observable because the coupling rate can be easily made rather large, $\Gamma_{0} \gtrsim 100 \mu \mathrm{eV}$.

In conclusion, we have considered an exactly solvable model of a resonance level coupled to a metallic reservoir. We have considered the transient process in which the level and the reservoir are coupled at time $t=0$ and determined the time dependence of the average values of the reservoir energy, of the resonant level energy, and of the interaction energy in the strong-coupling regime. We have also found the statistical distributions of these energies.

\section{ACKNOWLEDGMENTS}

We are glad to acknowledge very useful discussions with J. Pekola. B.D. was supported by DOMAST. D.G. was supported by the Academy of Finland Centre of Excellence program (Project No. 312057).
[1] J. P. Pekola, P. Solinas, A. Shnirman, and D. V. Averin, New J. Phys. 15, 115006 (2013).

[2] S. Suomela, A. Kutvonen, and T. Ala-Nissila, Phys. Rev. E 93, 062106 (2016).

[3] B. Donvil, P. Muratore-Ginanneschi, J. P. Pekola, and K. Schwieger, Phys. Rev. A 97, 052107 (2018).

[4] J. P. Pekola and B. Karimi, J. Low Temp. Phys. 191, 373 (2018).

[5] B. Donvil, P. Muratore-Ginanneschi, and J. P. Pekola, Phys. Rev. A 99, 042127 (2019).

[6] B. Karimi and J. P. Pekola, Phys. Rev. Lett. 124, 170601 (2020).

[7] R. Bulla, T. A. Costi, and T. Pruschke, Rev. Mod. Phys. 80, 395 (2008).

[8] H. T. Quan, Y. Liu, C. P. Sun, and F. Nori, Phys. Rev. E 76, 031105 (2007).

[9] J. Roßnagel, S. T. Dawkins, K. N. Tolazzi, O. Abah, E. Lutz, F. Schmidt-Kaler, and K. Singer, Science 352, 325 (2016).

[10] G. Benenti, G. Casati, K. Saito, and R. S. Whitney, Phys. Rep. 694, 1 (2017).

[11] M. Esposito, U. Harbola, and S. Mukamel, Rev. Mod. Phys. 81, 1665 (2009).

[12] M. Campisi, P. Hänggi, and P. Talkner, Rev. Mod. Phys. 83, 771 (2011).

[13] U. Seifert, Rep. Prog. Phys. 75, 126001 (2012).

[14] D. Collin, F. Ritort, C. Jarzynski, S. B. Smith, I. Tinoco, Jr., and C. Bustamante, Nature (London) 437, 231 (2005).

[15] S. Schuler, T. Speck, C. Tietz, J. Wrachtrup, and U. Seifert, Phys. Rev. Lett. 94, 180602 (2005).

[16] T. B. Batalhao, A. M. Souza, L. Mazzola, R. Auccaise, R. S. Sarthour, I. S. Oliveira, J. Goold, G. De Chiara, M. Paternostro, and R. M. Serra, Phys. Rev. Lett. 113, 140601 (2014).

[17] R. Kosloff, Entropy 15, 2100 (2013).

[18] S. Gasparinetti, P. Solinas, A. Braggio, and M. Sassetti, New J. Phys. 16, 115001 (2014).

[19] P. Wollfarth, A. Shnirman, and Y. Utsumi, Phys. Rev. B 90, 165411 (2014).

[20] A. Garg, J. N. Onichic, and V. Ambegaokar, J. Chem. Phys. 83, 4491 (1985).

[21] M. Toss, H. Wang, and W. H. Miller, J. Chem. Phys. 115, 2991 (2001).
[22] T. Le Goff, P. Politi, and O. Pierre-Louis, Phys. Rev. E 90, 032114 (2014).

[23] P. Strasberg, G. Schaller, N. Lambert, and T. Brandes, New J. Phys. 18, 073007 (2016).

[24] D. Newman, F. Mintert, and A. Nazir, Phys. Rev. E 95, 032139 (2017).

[25] P. Strasberg, G. Schaller, T. L. Schmidt, and M. Esposito, Phys. Rev. B 97, 205405 (2018).

[26] R. Martinazzo, B. Vacchini, K. H. Hughes, and I. Burghardt, J. Chem. Phys. 134, 011101 (2011).

[27] M. P. Woods, R. Groux, A. W. Chin, S. F. Huelga, and M. B. Plenio, J. Math. Phys. 55, 032101 (2014).

[28] M. Carrega, P. Solinas, M. Sassetti, and U. Weiss, Phys. Rev. Lett. 116, 240403 (2016).

[29] L. M. Cangemi, V. Cataudella, M. Sassetti, and G. De Filippis, Phys. Rev. B 100, 014301 (2019).

[30] J. Ankerhold and J. P. Pekola, Phys. Rev. B 90, 075421 (2014).

[31] A. Komnik, Phys. Rev. B 79, 245102 (2009).

[32] C. Cohen-Tannoudji, J. Dupont-Roc, and G. Grynberg, in AtomPhoton Interactions (Wiley, New York, 1998), pp. 165-255.

[33] P. Facchi, and S. Pascazio, Phys. Lett. A 241, 139 (1998).

[34] V. Jakšić, E. Kritchevski, and C. A. Pillet, in Large Coulomb Systems: Lecture Notes on Mathematical Aspects of QED, Lecture Notes in Physics Vol. 695 (Springer, Berlin, 2006), pp. 147-218.

[35] M. F. Ludovico, J. S. Lim, M. Moskalets, L. Arrachea, and D. Sanchez, Phys. Rev. B 89, 161306(R) (2014).

[36] M. Esposito, M. A. Ochoa, and M. Galperin, Phys. Rev. Lett. 114, 080602 (2015).

[37] A. Bruch, M. Thomas, S. Viola Kusminskiy, F. von Oppen, and A. Nitzan, Phys. Rev. B 93, 115318 (2016).

[38] P. Haughian, M. Esposito, and T. L. Schmidt, Phys. Rev. B 97, 085435 (2018).

[39] A. Oz, O. Hod, and A. Nitzan, J. Chem. Theory Comput. 16, 1232 (2020).

[40] M. A. Ochoa, A. Bruch, and A. Nitzan, Phys. Rev. B 94, 035420 (2016).

[41] F. Guinea, V. Hakim, and A. Muramatsu, Phys. Rev. B 32, 4410 (1985). 
[42] V. Jakšić, Y. Ogata, Y. Pautrat, and C.-A. Pillet, in Quantum Theory from Small to Large Scales (Oxford University Press, 2012), pp. 213-410.

[43] L. S. Levitov, in Quantum Noise in Mesoscopic Systems, edited by Yu. V. Nazarov (Kluwer, Dordrecht, 2003), pp. 373-396.

[44] I. Klich, in Quantum Noise in Mesoscopic Systems, edited by Yu. V. Nazarov (Kluwer, Dordrecht, 2003), pp. 397-402.

[45] T. Wolkanowski, arXiv:1303.4657.

[46] A. Rivas, A. D. K. Plato, S. F. Huelga, and M. B. Plenio, New J. Phys. 12, 113032 (2010).
[47] L. A. Khalfin, Sov. Phys. Dokl. 2, 340 (1957).

[48] H. D. Cornean, V. Moldoveanu, and C.-A. Pillet, Commun. Math. Phys. 331, 261 (2014).

[49] L. B. Wang, O.-P. Saira, D. S. Golubev, and J. P. Pekola, Phys. Rev. Appl. 12, 024051 (2019).

[50] H. Pothier, S. Guéron, N. O. Birge, D. Esteve, and M. H. Devoret, Phys. Rev. Lett. 79, 3490 (1997).

[51] Y. Niimi, Y. Baines, T. Capron, D. Mailly, F.-Y. Lo, A. D. Wieck, T. Meunier, L. Saminadayar, and C. Bäuerle, Phys. Rev. B 81, 245306 (2010). 